\title{
A Fully Printed CNT Based Humidity Sensor on Flexible PET Substrate
}

\author{
V. S. Turkani", B. B. Narakathu, D. Maddipatla, B. J. Bazuin, M. Z. Atashbar \\ Department of Electrical and Computer Engineering, \\ Western Michigan University, Kalamazoo, 1903 W. Michigan Avenue, Kalamazoo, Michigan, USA \\ vikramshreeshail.turkani@wmich.edu
}

\begin{abstract}
:
A fully printed carbon nanotube (CNT) based humidity sensor was developed for humidity sensing applications. The multi-layer humidity sensor was fabricated using additive print manufacturing processes on a flexible polyethylene terephthalate (PET) substrate. Two silver (Ag) electrodes were deposited using screen printing process. Gravure printing process was used to deposit a carbon nanotube (CNT) sensing layer between the Ag electrodes. The performance of the fabricated sensor was investigated by measuring its response towards varying relative humidity $(\% \mathrm{RH})$ ranging from $20 \% \mathrm{RH}$ to $80 \% \mathrm{RH}$, in steps of $10 \% \mathrm{RH}$. The resistive response of the humidity sensor exhibited a linear response over the entire operational $\mathrm{RH}$ range resulting in an overall resistance change of $6 \%$. In addition, a sensitivity of $0.1 \% / \% \mathrm{RH}$ and a correlation coefficient 0.9484 were calculated for the printed humidity sensor.
\end{abstract}

Key words: Printed Humidity Sensor, Screen Printing, Gravure Printing, Carbon Nanotube.

\section{Introduction}

In recent years, research has focused on the development of novel sensors for monitoring humidity which is one of the widely monitored parameter in the environmental, medical and food industries. Humidity sensors are typically fabricated on rigid substrates using conventional lithography processes which are often expensive [1]. Printed electronics, an emerging field for manufacturing cost-effective electronic devices on flexible platforms have been used to fabricate sensors such as pressure sensors, strain gauges, and humidity sensors [2-4]. Although hydrophilic polymers such as polymethyl methacrylate (PMMA), cellulose acetate butyrate (CAB), and poly (2hydroxyethyl methacrylate) (pHEMA) have been used as humidity sensing layers, changes in their permittivity cause relatively large drifts in the base value of the sensors, which demands frequent calibration of the sensors [4,5]. Carbon nanotubes (CNTs) have been used for chemical and biochemical sensing applications due to its large surface to volume ratio properties. [6-7]. However, there are relatively few reports on employing additive printing processes to develop CNT based humidity sensors. Therefore, there is a need to develop novel humidity sensors, which employ CNT based sensing layers, using additive printing processes.

\section{Experimental}

A schematic of the humidity sensor $(14 \times 8 \mathrm{~mm})$ is shown in Fig. 1(a). A flexible PET film (Melinex ${ }^{\circledR}$ ST730, $127 \mu \mathrm{m}$ thick), from DuPont Teijin Films was used as the substrate. A conductive silver (Ag) ink (AG-800, Applied Ink Solutions) was used to deposit two $\mathrm{Ag}$ electrodes on the substrate using an MSP 485 screen printer (Affiliated Manufacturing Inc.). A laboratory scale gravure press (K-Printing Proofer) with an electromechanically engraved gravure plate was employed for depositing the CNT ink, between the Ag electrodes. Fig. 1(b) shows the fully printed humidity sensor. The printed sensor was placed in a Thermotron ${ }^{\circledR}$ SE-3000-1-1 environmental chamber for investigating its electrical response. The resistive response of the sensor was measured using an Agilent E4980A precision LCR meter.

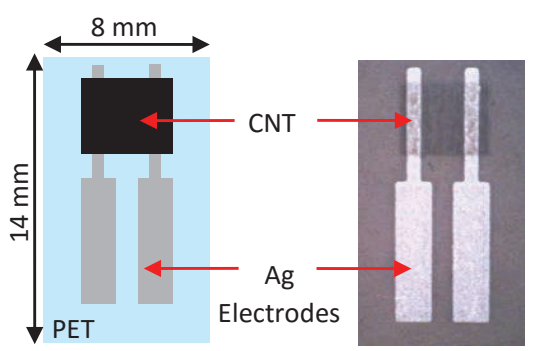

(a)

(b)

Fig. 1. a) Schematic of the humidity sensor and b) fully printed humidity sensor. 


\section{Results and Discussion}

Figure 2(a) shows the resistive response of the printed sensor towards varying $\mathrm{RH}$. The humidity of the chamber was varied form $20 \% \mathrm{RH}$ to $80 \% \mathrm{RH}$ during the humidification process and from $80 \% \mathrm{RH}$ to $20 \% \mathrm{RH}$ during the de-humidification process, in steps of $10 \%$ $\mathrm{RH}$ at a constant temperature $25^{\circ} \mathrm{C}$. The response of the sensor demonstrated a linear increase in resistance during the humidification process and recovered back to its base resistance, during the de-humidification process. The printed sensor demonstrated a maximum resistance change of $6 \%$ at $80 \% \mathrm{RH}$, when compared to its base resistance at $20 \% \mathrm{RH}$. The change in resistance can be attributed to the increase in the adsorption of the water molecules on to the surface of the CNT network when there is an increase in the humidity level. This adsorption results in electron transfer and depletes the majority charge carries (holes) in the CNT network [6]. The sensitivity (S) of the sensor was calculated to be $0.1 \% / \% \mathrm{RH}$ using Eq. (1) [6]:

$$
S=\left(\left(1 / R_{0}\right) *(\Delta R / \Delta \% R H)\right) * 100 \%
$$

where, $R_{0}$ is the base resistance at $20 \% \mathrm{RH}$, and $\Delta R / \Delta \% R H$ is the slope $(442.8 \Omega / \% \mathrm{RH})$ of the linear curve.

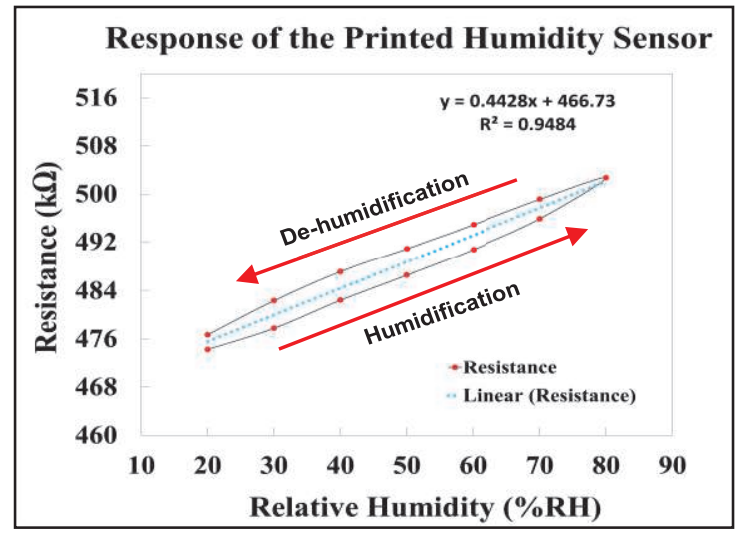

(a)

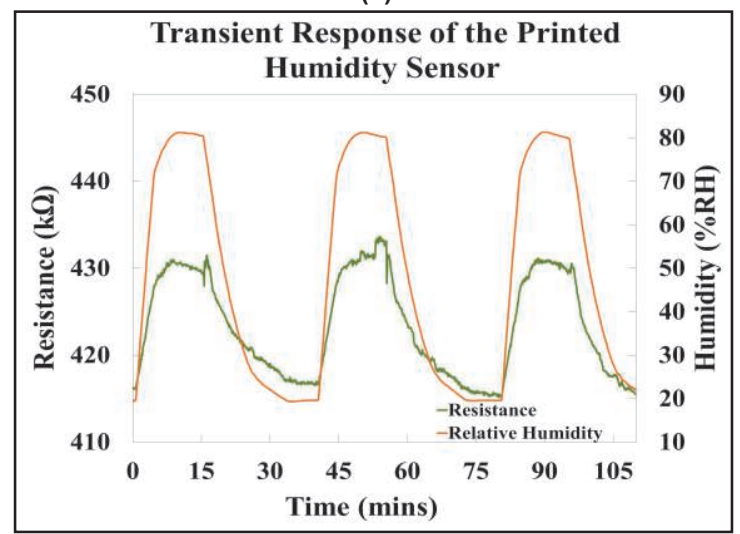

(b)

Fig. 2. (a) Resistive and (b) transient response of the printed humidity sensor.
Figure 2(b) shows the transient response of the printed sensor towards dynamic adsorptiondesorption cycles. The relative humidity in the chamber was interchanged between $20 \% \mathrm{RH}$ and $80 \% \mathrm{RH}$, at constant temperature of $60{ }^{\circ} \mathrm{C}$, and the response of the printed sensor followed the interchangeability with increase and decrease in the resistance values thus demonstrating the repeatability of the sensor at a relatively higher temperature.

\section{Conclusion and Future work}

A carbon nanotube (CNT) based humidity sensor was successfully fabricated using additive printing processes. Screen printing was employed to deposit Ag electrodes on a flexible PET substrate, while a CNT sensing layer was deposited using gravure printing between the electrodes. The response of the printed humidity sensor demonstrated a linear relationship towards relative humidity varying from $20 \% \mathrm{RH}$ to $80 \% \mathrm{RH}$, at a constant temperature of $25{ }^{\circ} \mathrm{C}$. An overall resistance change of $6 \%$ was observed. The sensitivity of the sensor was calculated to be $0.1 \% / \% \mathrm{RH}$ with a correlation coefficient of 0.9484 . The repeatability of the senor was demonstrated by measuring its transient response during dynamic adsorption-desorption cycles. Further research is underway to improve the sensitivity of the sensor by using CNT/polymer nanocomposites and investigating the effects of the bending on the performance of the sensor.

\section{References}

[1] S. Ali et al., All-printed humidity sensor based on graphene/methyl-red composite with high sensitivity, Carbon, 105, 23-32 (2016); doi: 10.1016/j.carbon.2016.04.013

[2] D. Maddipatla et al., A novel flexographic printed strain gauge on paper platform, In IEEE SENSORS, 1-4 (2015); doi: 10.1109/ICSENS.2015.7370606

[3] B. B. Narakathu et al., A novel fully printed and flexible capacitive pressure sensor, In Sensors, IEEE, 1-4 (2012); doi: 10.1109/ICSENS.2012.6411354

[4] A. S. G. Reddy et al., Fully printed flexible humidity sensor. $25^{\text {th }}$ Procedia Engineering, 120 123 (2011); doi: 10.1016/j.proeng.2011.12.030

[5] J. Lee et al., A resistive-type sensor based on flexible multi-walled carbon nanotubes and polyacrylic acid composite films, Solid-State Electronics, 87, 80-84 (2013); doi: 10.1016/j.sse.2013.05.001

[6] M. Z. Atashbar et al., Carbon nanotube networkbased biomolecule detection, IEEE Sensors; 524 - 528 (2006) doi: 10.1109/JSEN.2006.874491

[7] L. Liu et al., Humidity sensitivity of multi-walled carbon nanotube networks deposited by dielectrophoresis, Sensors, 9(3) 17141721(2009); doi:10.3390/s90301714 